Nazhat Shirzad-Wasei*, Jenny van Oostrum, Petra H.M. Bovee-Geurts, Lisanne J.A. Kusters, Giel J.C.G.M. Bosman and Willem J. DeGrip*

\title{
Rapid transfer of overexpressed integral membrane protein from the host membrane into soluble lipid nanodiscs without previous purification
}

\begin{abstract}
Structural and functional characterization of integral membrane proteins in a bilayer environment is strongly hampered by the requirement of detergents for solubilization and subsequent purification, as detergents commonly affect their structure and/or activity. Here, we describe a rapid procedure with minimal exposure to detergent to directly assemble an overexpressed integral membrane protein into soluble lipid nanodiscs prior to purification. This is exemplified with recombinant histagged rhodopsin, which is rapidly extracted from its host membrane and directly assembled into membrane scaffold protein (MSP) nanodiscs. We further demonstrate that, even when the MSP was his-tagged as well, partial purification of the rhodopsin-nanodiscs could be achieved exploiting immobilized-metal chromatography. Recoveries of rhodopsin up to $80 \%$ were achieved in the purified nanodisc fraction. Over $95 \%$ of contaminating membrane protein and his-tagged MSP could be removed from the rhodopsin-nanodiscs using a single $\mathrm{Ni}^{2+}$-affinity chromatography step. This level of purification is amply sufficient for functional studies. We provide evidence that the obtained rhodopsin-nanodisc preparations are fully functional both photochemically and in their ability to bind the cognate G-protein.
\end{abstract}

Keywords: baculovirus expression system; his-tagged rhodopsin; membrane protein solubilization; membrane scaffold protein; protein stabilization; recombinant protein.

\footnotetext{
*Corresponding authors: Nazhat Shirzad-Wasei and Willem J. DeGrip, Department of Biochemistry, 286-Radboud University Medical Center, Radboud Institute for Molecular Life Sciences, P.O. Box 9101, NL-6500 HB Nijmegen, The Netherlands, e-mail: nazhatshirzad@gmail.com; Wim.deGrip@radboudumc.nl Jenny van Oostrum, Petra H.M. Bovee-Geurts, Lisanne J.A. Kusters and Giel J.C.G.M. Bosman: Department of Biochemistry, 286-Radboud University Medical Center, Radboud Institute for Molecular Life Sciences, P.O. Box 9101, NL-6500 HB Nijmegen, The Netherlands
}

DOI 10.1515/hsz-2015-0100

Received January 3, 2015; accepted February 27, 2015; previously published online March 10, 2015

\section{Introduction}

Membrane proteins are involved in numerous biological processes, such as transport, signal transduction, and a variety of metabolic pathways. Up to $30 \%$ of the human proteome consists of integral membrane proteins (Wallin and VonHeijne, 1998), and these account for approximately half of all currently marketed therapeutic targets (Wishart et al., 2006). Despite their crucial biological relevance, relatively little is known in detail about their molecular mechanism and interaction patterns. An important bottleneck is the requirement of membrane proteins for a native-like membrane environment to maintain their correct structural folding and flexibility, and thereby, their physiological function.

Unfortunately, for most membrane proteins, studies of structure and function are limited due to either low expression levels and/or the absence of a simple and reproducible procedure for isolating these proteins in a monodisperse form that maintains their native structure. In the first purification step, membrane proteins commonly undergo solubilization with detergents, which nearly always destabilize these proteins and strongly affect their activity (Gohon and Popot, 2003; Zhou and Cross, 2013; Zoonens et al., 2013). The micellar environment, although amphipathic, will by itself destabilize the protein structure. Further, specific lipids that are essential for stability and/or activity may be lost during purification (DeGrip, 1982; DeGrip and Daemen, 1982; Rosenbusch, 2001).

Recently, studies have emerged that use small styrene-malic acid copolymers (SMA) for extracting integral membrane proteins from their natural cellular membrane. This eliminates a detergent solubilization step (Jamshad 
et al., 2011; Orwick-Rydmark et al., 2012; Long et al., 2013). The resulting complexes retain functional properties and they can be readily purified by affinity chromatography. However, this approach has a restricted $\mathrm{pH}$ range, the copolymer is highly negatively charged and the membrane protein is surrounded by only a limited number of lipid molecules (Gulati et al., 2014; Swainsbury et al., 2014).

Here, we present an alternative versatile approach exploiting the potential of 'membrane scaffold protein' (MSP) nanodiscs. This allows the extraction of integral membrane proteins from cellular membranes under minimal exposure to detergent, and to purify them in a preferentially monodisperse, stable state that maintains an amphipathic environment optimally preserving their structure and activity.

Over the last decade, elegant technology has been developed reconstituting integral membrane proteins into a soluble discoidal phospholipid bilayer environment confined by two copies of a genetically engineered MSP, termed nanodiscs (Bayburt et al., 2002, 2006; Ritchie et al., 2009; Marty et al., 2013). These nanodiscs have diameters ranging from $9.5 \mathrm{~nm}$ (nanodiscs) to $30 \mathrm{~nm}$ (macrodiscs) (Chromy et al., 2007; Park et al., 2011) and provide a native-like environment surrounding the hydrophobic domains of the membrane proteins. They exhibit high stability in a detergent-free aqueous solution and allow standard chromatographical procedures for protein purification (Bayburt et al., 2007; Whorton et al., 2007; Banerjee et al., 2008; Blanchette et al., 2009; Ritchie et al., 2009). The nanodisc technology has enabled biochemical and biophysical studies of a variety of membrane proteins (Bayburt et al., 2006, 2007; Boldog et al., 2006; Leitz et al., 2006; Whorton et al., 2007; Banerjee et al., 2008; Pandit et al., 2011), and membrane bound enzymes (Civjan et al., 2003; Denisov and Sligar, 2011). However, in almost all studies, the target proteins were purified using detergents before incorporation into nanodiscs. Only in a single early study, $N$-terminally anchored CYP was assembled into nanodiscs prior to purification (Civjan et al., 2003).

In this report, we describe the first study in which an integral membrane protein is extracted from its host membrane and directly assembled into MSP nanodiscs. The nanodiscs with the target protein can subsequently be purified, for example, by affinity chromatography (Figure 1B). This strongly minimizes exposure to detergents, and the resulting preparation retains full functional activity. The particular protein we selected is rhodopsin, the visual pigment of the vertebrate rod photoreceptor cell, an established paradigm for the $\mathrm{G}$ proteincoupled receptor (GPCR) family. Rhodopsin folds into the membrane-spanning seven-helical bundle typical of GPCRs. Its ligand and chromophore, 11-cis retinal, is covalently bound to Lys-296 in helix VII via a protonated Schiff-base linkage (Wald, 1968; Palczewski et al., 2000).

The presented approach is amenable to any membrane protein, and in particular useful for those proteins that are susceptible to perturbation by detergents. We further demonstrate that, even when his-tagged MSPs are employed for nanodisc formation, a single immobilized metal affinity chromatography (IMAC) purification step can already isolate the his-tagged target-protein containing nanodiscs from most of the contaminating nanodisc population.

\section{Results}

\section{Nanodisc formation: strategy}

Whereas the lipid bilayer in a nanodisc presents an appropriate environment for membrane proteins, the targetprotein is routinely solubilized and it is purified using a suitable detergent prior to incorporation into nanodiscs (Figure 1A). However, detergent solubilization and lipid removal seriously compromise structural and functional integrity of membrane proteins (Gohon and Popot, 2003; Zhou and Cross, 2013; Zoonens et al., 2013). These negative effects often cannot be fully repaired upon reconstitution of the purified protein within a bilayer environment. Here we propose an approach to minimize this problem by incorporating membrane proteins into nanodiscs immediately after brief solubilization of the cellular membranes with detergent. This has the major advantage that the target protein is only briefly exposed to detergents and that stabilizing lipids remain present. The nanodiscs are assembled with the available lipid pool in the cellular membranes used for heterologous expression, creating a system that closely mimics a natural membrane environment. We expect that this should provide sufficient lipids, since the lipid to protein ratio in natural membranes is close to 1:1 (w/w) and large proteins and complexes will not easily incorporate into nanodiscs. In fact, it has been recently demonstrated that nanodisc solubilization does represent the entire membrane proteome (Marty et al., 2013). Our approach is schematically explained in Figure 1B.

In order to model our approach, we used rhodopsin, as it can be easily monitored by its spectral properties and was shown before to retain full functionality in a nanodisc environment (Leitz et al., 2006; Bayburt 


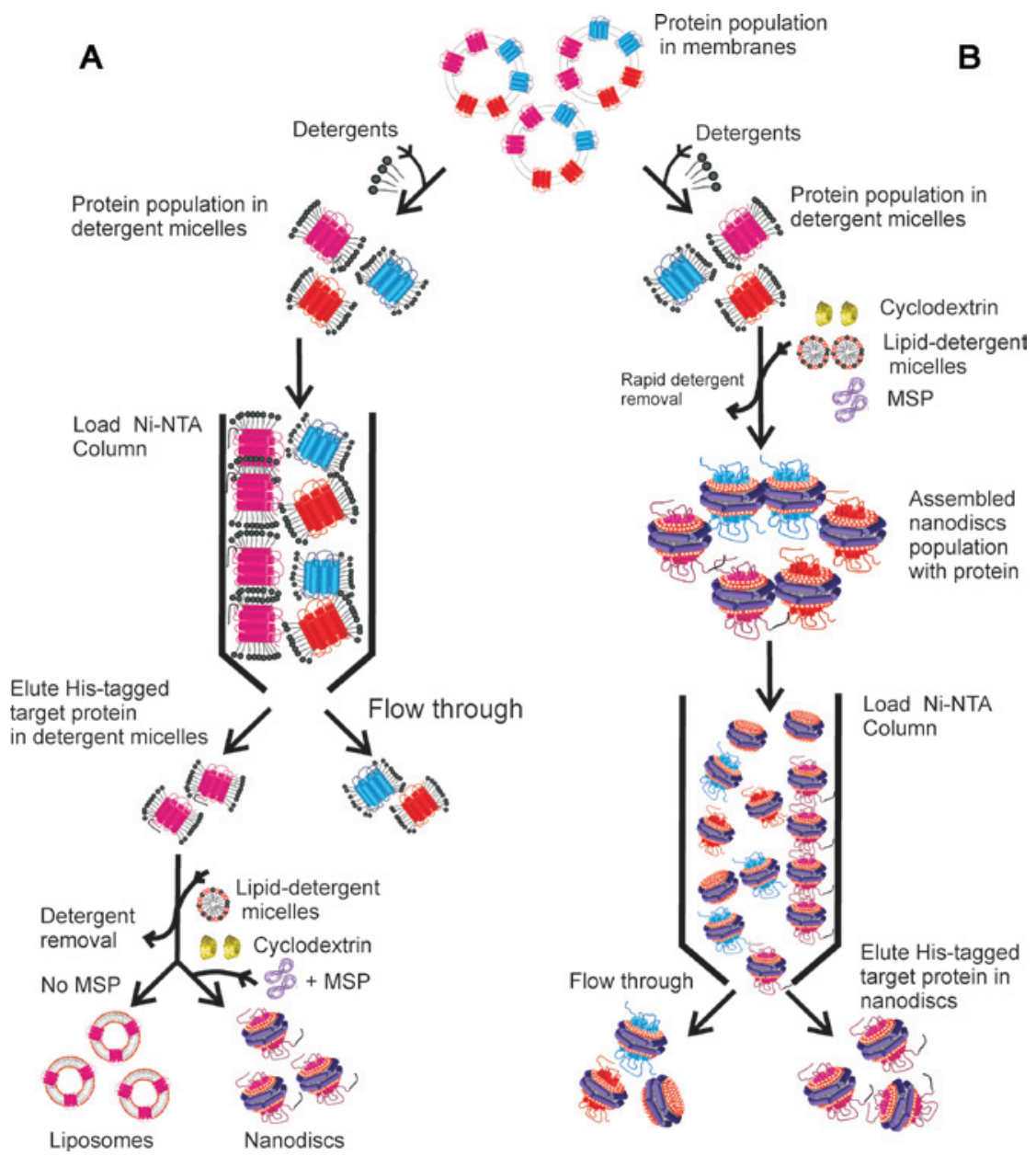

Figure 1: Schematic representation of our approach.

Compared are the classic approach (panel A) (target-protein incorporation into nanodiscs after purification) and our approach (panel B). In the latter, first, the entire membrane population is incorporated into nanodiscs and out of this total mixture, the target-protein nanodiscs are purified. Note that the liposomes that can be generated with the purified target-protein (panel A), in reality are much larger (diameter varying between 100 and $5000 \mathrm{~nm}$ ) than the nanodiscs (12-30 nm).

et al., 2007, 2011; Banerjee et al., 2008; Whorton et al., 2008; Tsukamoto et al., 2011). Native rhodopsin can be extracted from purified rod outer segment membranes (DeGrip et al., 1980). His-tagged opsin can be heterologously expressed in S $f 9$ insect cells (Janssen et al., 1995; Klaassen et al., 1999) and incubation with 11-cis retinal generates his-tagged rhodopsin. For the generation of nanodiscs we opted for the membrane scaffold protein zebrafish Apo A-1 (ZAP1), shown before to form functional nanodiscs with purified rhodopsin (Banerjee et al., 2008). To estimate the minimal quantity of ZAP1 required for proper nanodisc formation of the membrane protein population of $S f 9$ cells, we used a membrane protein content of $225 \pm 25 \mu \mathrm{g}$ per $10^{6}$ cells (DeCaluwé et al., 1993) and estimated the average mass of the membrane protein populationat $55 \pm 5 \mathrm{kDa}$ (Prioretal., 2011; Martyetal.,2013).
This results in approximately $4 \mathrm{nmol}$ of membrane protein per $10^{6}$ cells. For nanodisc assembly with purified membrane proteins, a minimal molar ratio of two MSP per membrane protein (MP) is required, but in practice, ratios of 2-25 have been employed (Bayburt et al., 2007; Banerjee et al., 2008; Ritchie et al., 2009; Pandit et al., 2011; Inagaki et al., 2013; Mors et al., 2013). A higher ratio may result in a more homogeneous population of MP-nanodiscs, but also generates larger contamination with empty nanodiscs and single MSPs. As the entire membrane protein population will not insert into nanodiscs, and to be cost-effective with respect to MSP, we considered a 2-3-fold molar excess of MSP, that is, $8-12 \mathrm{nmol}$ of MSP per $10^{6}$ cells. For solubilization of rhodopsin, we selected n-dodecyl$\beta$-D-maltoside (DDM), a detergent commonly used for 
membrane protein solubilization (Rigaud et al., 1998) and shown to be effective as well as mild towards rhodopsin (DeGrip, 1982). Detergent extraction to generate nanodiscs is usually accomplished by incubation with a lipophilic matrix (Rigaud et al., 1998; Civjan et al., 2003; Leitz et al., 2006; Bayburt et al., 2007; Banerjee et al., 2008). However, this is a relatively slow process and, in our hands, led to considerable loss of protein. To effectuate rapid detergent extraction with minimal loss of protein, we selected the cyclodextrin-inclusion procedure, which also affords quantitative removal of detergent, but allows better control over kinetics and time investment (DeGrip et al., 1998).

\section{Nanodisc formation: experimental implementation}

\section{Opsin expression, rhodopsin regeneration and solubilization}

Bovine opsin extended with a C-terminal deca-histidine tag was produced in $S f 9$ insect cells using recombinant baculovirus, as described (Klaassen et al., 1999; ShirzadWasei et al., 2013). Full-length opsin expression was confirmed by immunoblotting (Figure 2, lane 1). The triad pattern represents diglycosylated (upper band), mono glycosylated (middle band) and unglycosylated (bottom band) opsin (Janssen et al., 1991; DeCaluwé and DeGrip, 1996). To convert the apo-protein opsin into the holo-pigment rhodopsin, which allows for a straightforward spectroscopic assay (Wald, 1968; DeGrip, 1982), membranes from infected $\mathrm{S} f 9$ cells were incubated with 11-cis retinal. This reaction is completed at room temperature (RT) within $60 \mathrm{~min}$. Subsequently, hydroxylamine was added to $50 \mathrm{~mm}$ final concentration to convert excess retinal into the chemically more stable retinaloxime (Wald, 1968; Groenendijk et al., 1980). The expression level of rhodopsin can be assessed after solubilization from the absorbance and its molar absorbance coefficient at $500 \mathrm{~nm}$ [40 $600 \mathrm{M}^{-1} \mathrm{~cm}^{-1}$; (Wald, 1968; Daemen et al., 1970)]. To minimize solubilization time and exposure of the target protein to detergent prior to nanodisc formation, a relatively low membrane density was employed (equivalent to $20 \times 10^{6}$ cells $/ \mathrm{ml}$ ) in combination with a moderate detergent concentration (20 mM DDM). Near-complete solubilization of rhodopsin was achieved within $30 \mathrm{~min}$ at RT or within $60 \mathrm{~min}$ at $4^{\circ} \mathrm{C}$. Immunoblot analysis demonstrates that predominantly the diglycosylated upper band is solubilized (Figure 2, lane 2). This is in line with earlier conclusions that the majority of the mono- and the

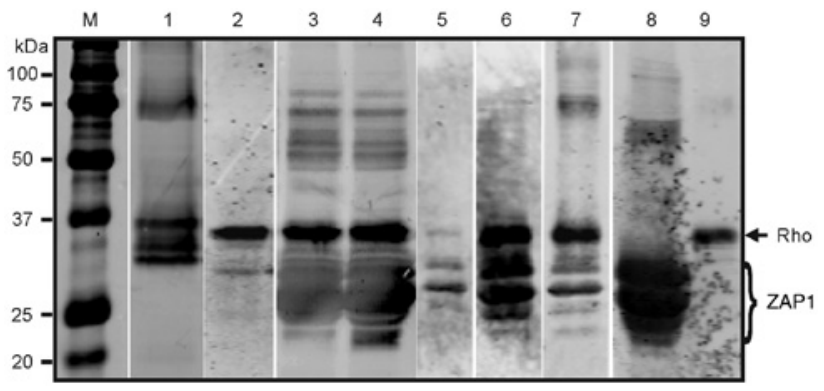

Figure 2: Immobilized metal affinity chromatography purification profile of rhodopsin-nanodiscs analyzed by immunoblotting.

The blot was screened with anti-his-tag antibody. Lane $M$ represents marker proteins with molecular weights indicated. Lane 1 shows the $S f 9$ cell membrane fraction (see the text for explanation of the triad pattern for opsin in the $30-40 \mathrm{kDa}$ range). Lane 2 contains the $\mathrm{n}$-dodecyl- $\beta$-D-maltoside extract of S $f 9$ membranes after regeneration of rhodopsin. The sample in lane 3 is taken after addition of the membrane scaffold protein zebrafish Apo A-1 (ZAP1) to the extract. Note the big blurred band below rhodopsin that represents the large excess of ZAP1. Lane 4 shows the supernatant after nanodisc formation and spinning down the insoluble components, confirming that soluble rhodopsin-nanodiscs have been generated. The majority of the protein contamination including ZAP1 elutes in the non-bound fraction and the additional washes with buffer and low concentrations of histidine (cf. Figure 3). Most of the remaining ZAP1 is eluted in the $50 \mathrm{~mm}$ histidine fraction (lane 5) where rhodopsin slowly starts to elute. The remaining rhodopsin co-elutes with the remaining ZAP1 in the $200 \mathrm{~mm}$ histidine fraction (lane 6) and the $270 \mathrm{~mm}$ histidine fraction (lane 7). Lanes 8 and 9 present purified ZAP1 and his-tagged rhodopsin, respectively. ZAP1 is expressed and purified in three major forms that are all competent in nanodisc formation (Pandit et al., 2011).

unglycosylated protein is not correctly folded and aggregates in the presence of a mild detergent (Janssen et al., 1991; DeCaluwé and DeGrip, 1996).

\section{Nanodisc assembly}

For assembly of a homogeneous population of nanodiscs, a defined ratio of phospholipids to MSP is recommended (Bayburt et al., 2002). We utilized the available lipid pool of cellular membranes and first tested this on bovine rod outer segment membranes, which mainly contain rhodop$\sin$ (DeGrip et al., 1980). After brief solubilization, addition of ZAP1 to a 3-fold molar excess over rhodopsin, formation of nanodiscs and centrifugation to precipitate larger structures, the supernatant was analyzed for the presence of rhodopsin by spectroscopy. As shown in Figure 3, over $90 \%$ of the rhodopsin was recovered in the soluble nanodisc fraction, indicating that the natural membrane lipid pool can be sufficient to accommodate the nanodiscs. 


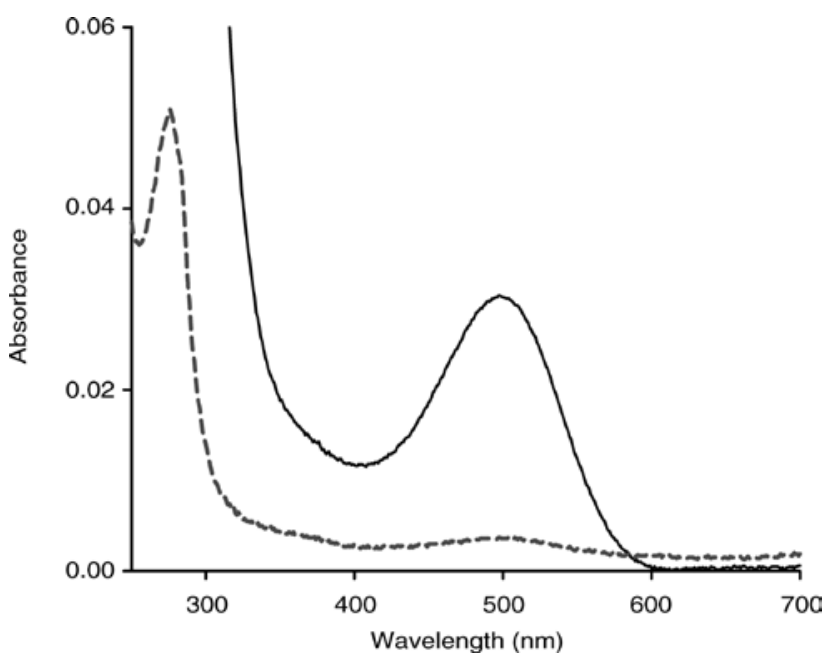

Figure 3: Rhodopsin-nanodisc formation from solubilized rod outer segment membranes.

Following nanodisc generation larger structures were precipitated and pellet and the supernatant analyzed by spectroscopy. The continuous curve displays the spectral properties of the supernatant, showing the typical absorbance band of rhodopsin around $500 \mathrm{~nm}$. The spectrum of the pellet, after solubilization in the same volume, is presented in the dashed curve. Over $90 \%$ of the rhodopsin is recovered in the soluble nanodisc fraction.

Subsequently, this was tested on his-tagged rhodopsin heterologously produced in $\mathrm{S} f \mathrm{i}$ insect cells, where rhodopsin constitutes $<0.5 \%$ of the membrane protein population (Klaassen et al., 1999; Bosman et al., 2003). In this case, lipid content and composition of the cellular membranes may vary somewhat over different cultures. We observed that under those conditions the recovery of rhodopsin in nanodiscs varied considerably. Instead, addition of extra lipid [lipid:MP ratio of 1:4 (w/w)] produced quite reproducible results. For the extra lipid, we selected asolectin, a natural lipid source that has a good variety in lipid species and fully maintains the functionality of a variety of membrane proteins, including rhodopsin (Sone et al., 1977; Klaassen and DeGrip, 2000; Ratnala et al., 2004). Asolectin could be added before or after solubilization of the membrane fraction.

Upon removal of detergent, nanodiscs self-assemble from a mixture of phospholipid/detergent mixed micelles and MSP (Bayburt et al., 2002). Many GPCRs show a timedependent loss of activity in detergents (Navratilova et al., 2005); hence, long-term exposure to detergents needs to be avoided. The cyclodextrin-inclusion procedure described earlier (DeGrip et al., 1998) allows rapid assembly of membrane proteins (MP), lipid and MSP into nanodiscs with good recovery of MP (Pandit et al., 2011). In the example presented here (Figure 2), we used ZAP1 as MSP (Banerjee et al., 2008) and an estimated 1:2 molar ratio of membrane protein to ZAP1. Similar results were obtained with the MSPs MSPD1 and MSPE3D1. Molar ratios of membrane protein to MSP of 1:3 yielded comparable recoveries. Generation of nanodiscs was initiated by addition of a slight excess of heptakis-2,6-di-O-methyl- $\beta$-cyclodextrin ( $\beta$-cyclodextrin), which will extract detergent from mixed micelles under formation of inclusion complexes (DeGrip et al., 1998). Addition in three steps allows gradual selfassembly of the nanodiscs. Even more rapid formation of nanodiscs, that is, within $15 \mathrm{~min}$, can be accomplished by single-step addition of $\beta$-cyclodextrin, but this may result in a lower recovery or a more heterogeneous population of nanodiscs (Pandit et al., 2011).

\section{Partial purification of rhodopsin-nanodiscs}

Following nanodisc assembly, insoluble contamination, such as protein aggregates and accidental proteoliposomes, was removed by centrifugation. The resulting supernatant contained empty and MP-nanodiscs, possibly excess MSP, cyclodextrin-detergent complexes, and excess cyclodextrin (Figure 2, lane 4). The most straightforward procedure to purify the target-protein nanodiscs from this mixture would be exploiting dedicated affinity chromatography, for example, using an immobilized antibody against the target protein or an immobilized ligand. This is well documented in the literature (Bayburt et al., 2007; Banerjee et al., 2008; Whorton et al., 2008; D’Antona et al., 2014) and does not need to be further demonstrated here. However, such options are not often available. A his-tag is the most popular tag for recombinant proteins, allowing easy, affordable and high-capacity purification over immobilized- $\mathrm{Ni}^{2+}$ or $\mathrm{Co}^{2+}$-matrices (Janssen et al., 1995; Klaassen et al., 1999). In our case, we anticipated that MSPs and other nanodiscs would be co-purified owing to the his-tag carried by the MSP, but we observed that empty nanodiscs assembled with his-tagged MSP (HT-ND) have a relatively low affinity for the $\mathrm{Ni}^{2+}$-matrix. This allows for a differential elution from the IMAC matrix of HT-ND containing his-tagged rhodopsin, empty HT-ND and HT-ND containing non-his-tagged membrane protein.

A typical example is presented in Figures 2 and 4. While most of the rhodopsin-nanodiscs are bound to the nickel matrix (Table 1), most of the protein contamination including the ZAP1 proteins appears in the flow-through and the wash (Figure 4, lane 2). Most of the remaining ZAP1 elutes in the $50 \mathrm{~mm}$ histidine fraction, where rhodopsin only slowly starts to elute (Figure 2, lane 5; Figure 4, lanes 5 and 6). Subsequent treatment with 200 and $270 \mathrm{~mm}$ histidine elutes the remaining rhodopsin, co-purifying with 


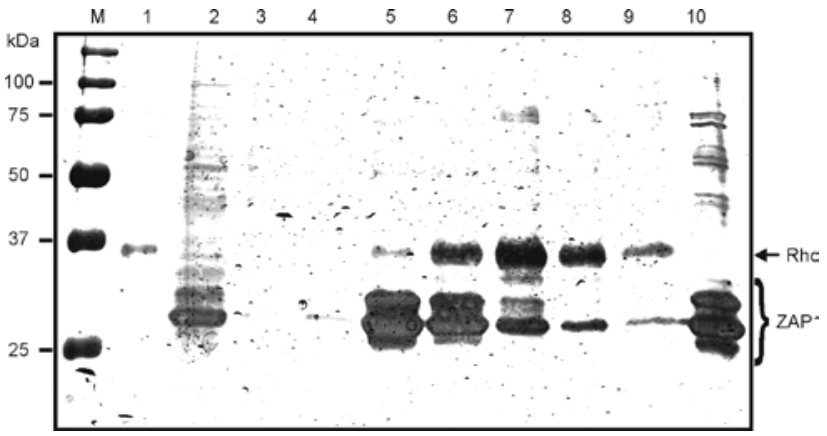

Figure 4: Immobilized metal affinity chromatography (IMAC) purification profile of rhodopsin-nanodiscs analyzed using silver staining.

Fractions were separated by SDS-PAGE, and analyzed for protein by silver staining. Membrane fractions, extracts, supernatant after nanodisc formation and IMAC flow through are not shown, as they are overabundant with $\mathrm{S} f 9$ membrane proteins. Lane $M$ represents marker proteins with molecular weight indicated. Lanes 1 and 10 represent purified his-tagged rhodopsin and purified zebrafish Apo A-1 (ZAP1), respectively. Lanes 2-4 present wash fractions showing that most of the protein contamination and part of the ZAP1 proteins are removed at that stage. Lanes 5 and 6 contain the $50 \mathrm{~mm}$ histidine elution fractions, demonstrating elution of most remaining contaminating proteins and ZAP1, and rhodopsin beginning to elute. Lanes 7 and 8 (200 mm histidine fractions) and lane 9 (270 $\mathrm{mm}$ histidine fraction) represent elution of rhodopsinnanodiscs, as rhodopsin and ZAP1 co-elute.

Table 1: Recovery of rhodopsin-nanodiscs.

\begin{tabular}{lrr}
\hline Fraction & \multicolumn{2}{c}{ Percentage rhodopsin } \\
\cline { 2 - 3 } & Immunoblot & Spectroscopy \\
\hline Membrane extract & $\equiv 100$ & $\equiv 100$ \\
Non-bound fraction & $11 \pm 7$ & n. d. \\
Wash fraction & $17 \pm 15$ & n. d. \\
Purified nanodiscs & $72 \pm 8$ & $55 \pm 8$ \\
\hline
\end{tabular}

The amount of rhodopsin solubilized in detergent is taken as $100 \%$. Immobilized metal affinity chromatography (IMAC) fractions are quantified with quantitative immunoblot analysis using primary anti-His and anti-rhodopsin antibodies in combination with the Odyssey imaging system (left column). Calibrated amounts of purified recombinant bovine his-tagged rhodopsin were applied as an internal standard (Klaassen et al., 1999). Rhodopsin-nanodiscs present in the purified IMAC fractions were also quantified by spectroscopy (right column). As immunoblotting is significantly more sensitive than spectroscopy, the latter probably underestimates the rhodopsin levels in the IMAC fractions. Values are given with SD, based upon three experiments; n.d., not detectable.

the remaining ZAP1 (Figure 2, lanes 6 and 7; Figure 4, lanes 7,8 , and 9). Thus, a single IMAC procedure can purify histagged rhodopsin-nanodiscs from the large majority of contaminating proteins and nanodiscs, even if the MSP is his-tagged as well. Preliminary evidence suggests that this purification can be fine-tuned by using a slightly concave continuous gradient of histidine (10-270 mM). We surmize that in the nanodiscs the his-tag is not sufficiently accessible or is structurally too restrained to bind with high affinity to the matrix-bound metal ion. Quantitative immunoblot analysis using primary anti-his and anti-rhodopsin antibodies revealed excellent recoveries of up to $80 \%$ of the rhodopsin solubilized from the cellular membranes (Table 1, left column). This is at least comparable to earlier results obtained via path A in Figure 1, where following reconstitution into proteoliposomes or nanodiscs after purification $40-70 \%$ of the originally solubilized rhodopsin is recovered (Klaassen et al., 1999; Klaassen and DeGrip, 2000; Mors et al., 2013).

Spectra of the purified rhodopsin-nanodisc fractions are characterized by three absorbance bands, peaking near 280, 365, and $500 \mathrm{~nm}$ (Figure 5). The absorbance at $280 \mathrm{~nm}$ represents the total protein population, including rhodopsin and ZAP1. The $365 \mathrm{~nm}$ band is due to retinaloxime, generated from hydroxylamine and excess retinal, which became inserted in the lipid phase of nanodiscs. Retinaloxime is not a ligand for opsin and does not affect the functionality of rhodopsin (Wald, 1968; Hubbard et al., 1971). The absorbance band around $500 \mathrm{~nm}$ is typical for rhodopsin. Upon illumination in the presence of hydroxylamine it is converted to a band at $365 \mathrm{~nm}$ (Figure 6), caused

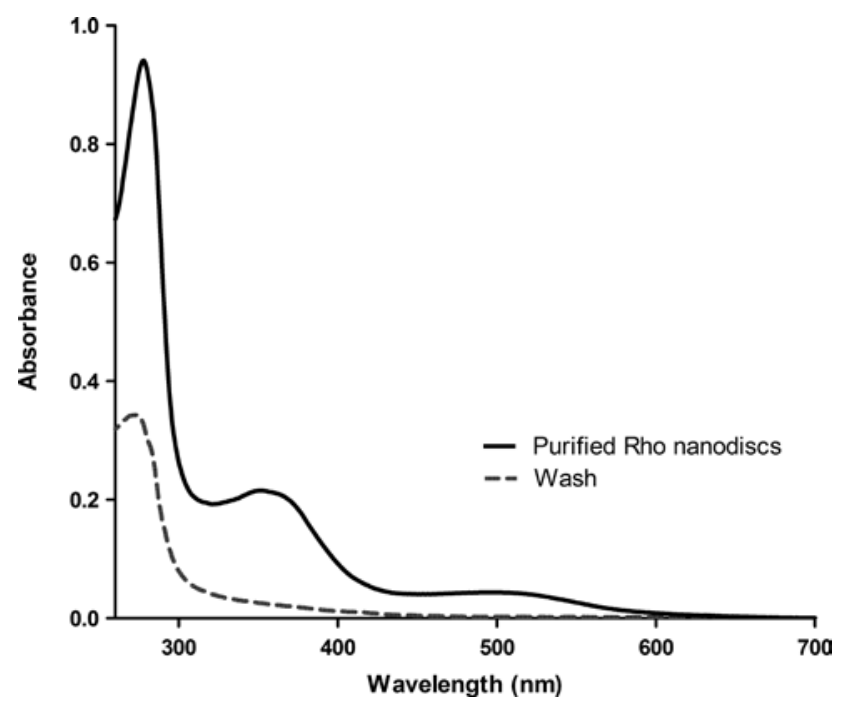

Figure 5: Spectral analysis of immobilized metal affinity chromatography purification fractions.

Typical UV/Vis absorbance spectra of a diluted wash fraction (dashed curve) mainly presenting a protein band at $280 \mathrm{~nm}$ and a purified rhodopsin-nanodisc fraction (continuous curve) displaying the additional typical rhodopsin band at $500 \mathrm{~nm}$ and excess ligandderived retinaloxime at $365 \mathrm{~nm}$. 
by conversion of all photoproducts to retinaloxime (Wald, 1968). We estimated the rhodopsin recovery in the IMAC fractions by spectroscopy. However, this assay has much lower sensitivity and did not detect rhodopsin in the flowthrough and wash fractions. Thus, the estimated recovery of rhodopsin in the purified nanodiscs was somewhat lower than that estimated by immunoblot (Table 1, right column).

The ratio A280/A500 in the spectra of the purified rhodopsin-nanodiscs allows us to estimate the efficacy of the purification procedure. For a highly purified rhodopsin-ZAP1-nanodisc preparation, this ratio was reported to be approximately 5 (Banerjee et al., 2008). In our partially purified rhodopsin-ZAP1-nanodisc samples, this ratio varies between 15 and 20, indicating that, as anticipated, we did not yet achieve high-level purification. Nevertheless, considering that in the host membranes recombinant rhodopsin accounts for maximally $0.5 \%$ $(\mathrm{w} / \mathrm{w})$ of the total MP population, and that MSP has been added in a 2-fold molar ratio to total MP, we estimate that over $95 \%$ of contaminating protein has been removed in a single IMAC step. This level of purification is more than sufficient for spectral, as well as functional studies, as we will demonstrate in the next section.

\section{Rhodopsin-nanodiscs: characterization}

\section{Dynamic light scattering: size distribution}

For empty ZAP1-nanodiscs, diameters of 10-12 $\mathrm{nm}$ have been measured, while protein-containing

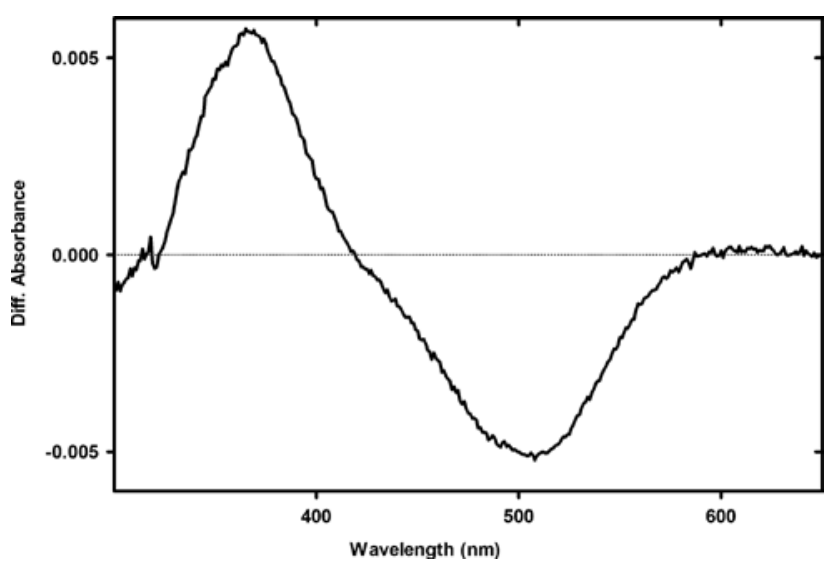

Figure 6: Typical difference spectrum demonstrating the photosensitivity of purified rhodopsin-nanodiscs.

The spectrum of purified rhodopsin-nanodiscs was subtracted from that obtained after illumination of the same sample in the presence of $50 \mathrm{~mm}$ hydroxylamine. It shows the typical loss of the rhodopsin absorbance band near $500 \mathrm{~nm}$ with generation of the retinaloxime band around $365 \mathrm{~nm}$.
ZAP1-nanodiscs vary in size between 12 and $17 \mathrm{~nm}$, probably depending on the type of assay and the lipid content (Banerjee et al., 2008). Dynamic light scattering analysis of our purified rhodopsin-nanodisc preparations yielded a bimodal distribution of a major component ( $\geq 98 \%$ in particle number) with a fairly narrow size distribution $(17 \pm 3 \mathrm{~nm})$ and a minor larger component $(82 \pm 7 \mathrm{~nm} ; \leq 2 \%$ in particle number). The size distribution of the smaller component agrees with a single nanodisc, while the larger component probably consists of a more composite build-up (Banerjee et al., 2008; Ritchie et al., 2009; Inagaki et al., 2013).

\section{Photochemical properties: $\mathrm{pH}-$ dependent photolysis}

In this and the following section, we show that our rhodopsin-nanodisc preparation not only is photosensitive, but also fully active in signal transmission. The rationale of this assay is described in the Supplementary Information. The photochemical integrity of rhodopsin in the nanodisc preparation was evaluated by photolysis at two $\mathrm{pH}$-values ( $\mathrm{pH} 6.0$ and $\mathrm{pH}$ 8.0). The spectral transitions observed in illuminated rhodopsin-nanodiscs at $10^{\circ} \mathrm{C}$ and $\mathrm{pH} 6.0$ are shown as two difference spectra (Figure 7A, curves 1 and 2). Curve 1 is obtained by subtracting the 'dark' spectrum from the spectrum recorded immediately after illumination. Curve 2 is obtained by subtracting the latter spectrum from that recorded 30 min after illumination. Curve 1 shows the immediate disappearance of rhodopsin upon illumination (negative band around $500 \mathrm{~nm}$ ), together with a slight positive inflection near $480 \mathrm{~nm}$ (Meta I) and a strong new band at $380 \mathrm{~nm}$ (Meta II). Subsequently, Meta II decays under release of retinal into several products (Figure 7A, curve 2), one of which is represented by an absorbance band peaking around $450 \mathrm{~nm}$ (VanBreugel et al., 1979). Thus, the rhodopsin-nanodisc preparation presents the established late-phase photocascade (Matthews et al., 1963; Emeis et al., 1982; Sato et al., 2010; Tsukamoto et al., 2011). The same conclusion can be drawn from a similar experiment at $\mathrm{pH}$ 8.0 (Figure 7B), where we expect Meta I and Meta III as the major late photoproducts, (Wald, 1968; Hofmann, 1986; Vogel et al., 2004). Indeed, curve 1 in Figure 7B presents a more red-shifted negative band near $525 \mathrm{~nm}$ (photolysed rhodopsin), because now the photoproducts Meta I (480 nm) and Meta III $(470 \mathrm{~nm})$ dominate and there is no evidence for formation of Meta II (380 nm). Subsequently, slow decay of Meta I into Meta III and free retinal $(380 \mathrm{~nm})$ is observed (Figure 7B, curve 2). 


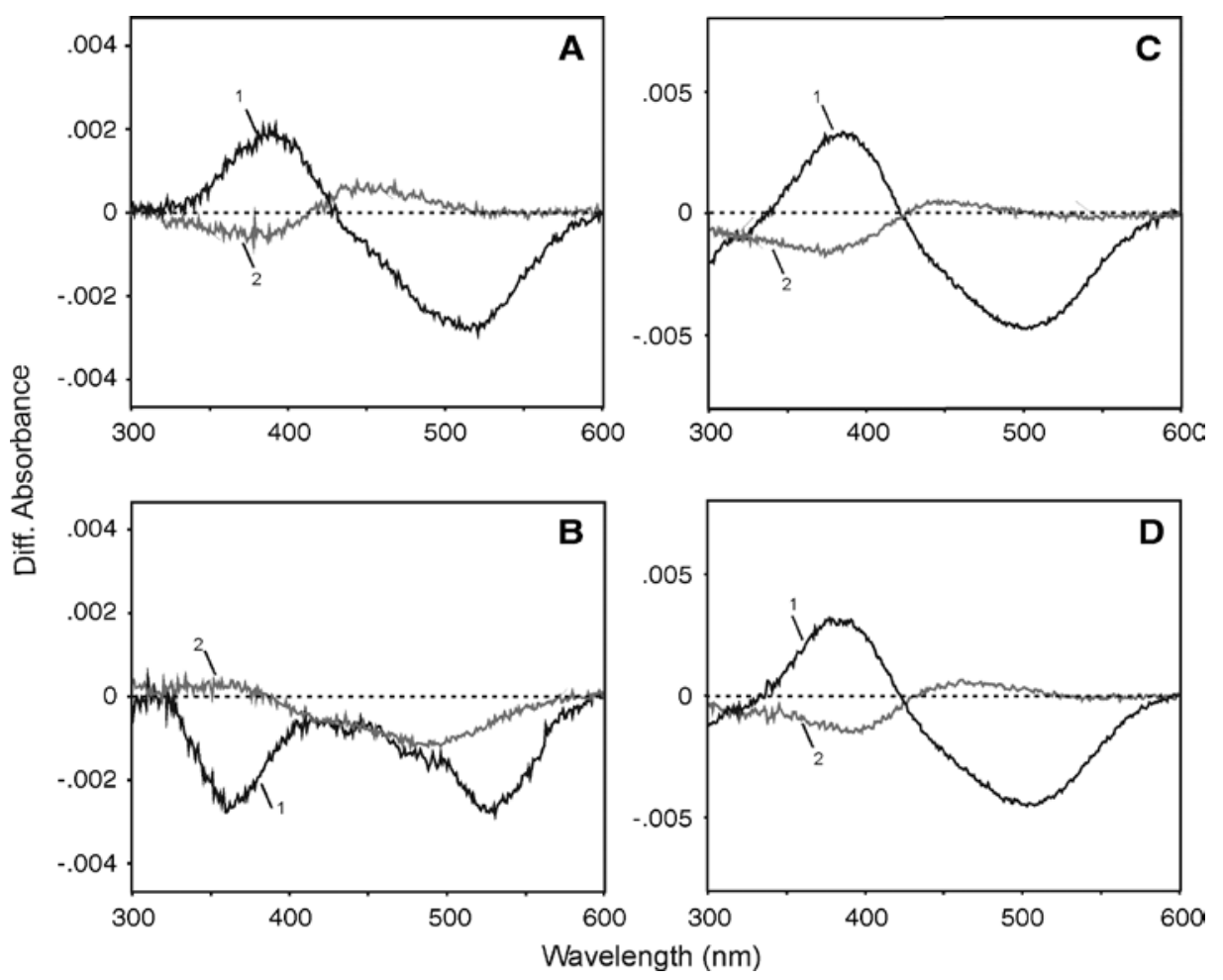

Figure 7: Late photointermediates and downstream signalling of purified rhodopsin-nanodiscs.

All spectra were recorded at $10^{\circ} \mathrm{C}$ (see the materials and methods section and the text for details of the protocol). The late transitions are best presented in difference spectra. In all panels, curve 1 and 2 are difference spectra of rhodopsin-nanodiscs and/or photoproducts generated by brief irradiation of rhodopsin with $>530 \mathrm{~nm}$ light at the indicated pH. Curve 1 is obtained by subtracting the 'dark' spectrum from the spectrum recorded $10 \mathrm{~s}$ after illumination. Curve 2 is obtained by subtracting the latter from the spectrum recorded at 30 min after illumination. Panel (A) presents the late transitions following illumination at pH 6.0. Curve 1 shows the typical loss of rhodopsin upon illumination (negative band near $500 \mathrm{~nm}$ ) with predominant formation of the active state Meta II (positive band around $380 \mathrm{~nm}$ ) and a minor amount of Meta I (positive indentation in the $500 \mathrm{~nm}$ band around $470 \mathrm{~nm}$ ). Curve 2 presents the subsequent decay of Meta II (negative band around $380 \mathrm{~nm}$ ) with concomitant release of ligand (retinal) that generates a random population of protonated Schiff bases (positive band around $450 \mathrm{~nm}$ ). Panel (B) presents the late transitions following illumination at $\mathrm{pH}$ 8.0. The negative band in curve 1 representing loss of rhodopsin has a strong positive indentation around $480 \mathrm{~nm}$, because of the predominant formation of Meta I (480 nm) with some Meta III $(470 \mathrm{~nm})$. The negative band at $360 \mathrm{~nm}$ is an alkaline $\mathrm{pH}$ artefact probably originating in the retained retinaloxime. Curve 2 now presents slow decay of Meta I into Meta III and free retinal (positive band around $360 \mathrm{~nm}$ ). Panel (C) presents the same condition as panel (A) $(\mathrm{pH}$ 6.0), except that $20 \mu \mathrm{M}$ of the Gt $\alpha$-C-terminal peptide analog is present. This strongly binds to Meta II, pushing the Meta I $\leftrightarrow$ Meta II equilibrium entirely to the Meta II state (see Supplementary Information). The overall pattern in panel (C) is very similar to that in panel (A), except that a larger amount of Meta II is generated and that there is no indication for the presence of Meta I. The peptide does not inhibit decay of Meta II, as signified by the release of retinal (curve 2). Panel (D) presents the same condition as panel (B) (pH 8.0), except with $20 \mu \mathrm{M}$ of the Gt $\alpha$-C-terminal peptide analog present. The pattern is now completely similar to that at pH 6.0 (panel C), demonstrating that even at $\mathrm{pH} 8.0$, binding of the peptide to Meta II completely shifts the equilibrium to the Meta II side.

\section{G-protein interaction: effect of a transducin peptide on rhodopsin photolysis}

We next examined whether the Meta II photoproduct in our nanodisc preparation is able to bind its cognate heterotrimeric GTP binding protein, transducin (Gt). The principle again is explained in the Supplementary Information. For convenience, we employed an analog of the C-terminal peptide of the transducin $\alpha$-subunit $\left(\mathrm{NH}_{2}\right.$-VLEDLKSCGLF-COOH) that has high affinity for Meta II (Aris et al., 2001; Sato et al., 2010). Photolysis of the rhodopsin-nanodisc preparation at $\mathrm{pH} 6.0$ in the presence of this peptide clearly enhances the formation of Meta II, and there is no longer evidence for the presence of Meta I (Figure 7C, curve 1). The relative amount of Meta II generated in the absence of peptide varies between $60 \%$ and $70 \%$ of that in the presence of peptide, which is in good agreement with a pKa of $6.5 \pm 0.2$ (see Supplementary Information). The peptide does not influence the subsequent decay pattern of Meta II; the same patterns are observed with and without the peptide (curve 2 in Figure 7A and C). 
Even more importantly, photolysis at $\mathrm{pH} 8.0$ in the presence of peptide completely matches the data at $\mathrm{pH} 6.0$ with peptide, that is, single formation of Meta II (Figure 7D, curve 1). Hence, even at $\mathrm{pH} 8.0$ binding of the peptide to Meta II completely shifts the equilibrium to the Meta II side, and the subsequent decay follows the same pattern as that at pH 6.0 (Figure 7D, curve 2).

\section{Discussion}

Various procedures have been developed to study membrane proteins in vitro using detergents and/or lipidbased systems. It has gradually become apparent that the natural phospholipid bilayer presents the optimal micro-environment to investigate the physiological function and structure of membrane proteins (Silvius, 1992; Popot, 2010). For such studies, membrane proteins have to be properly purified. However, for unrestricted extraction of membrane proteins from their membrane environment, detergents have proven to be indispensable. Here, we describe an approach to directly transfer the targetprotein from the cellular membrane into MSP nanodiscs, employing the natural lipid pool and requiring only short exposure to detergent. The target-protein nanodiscs can then be selectively purified from the resulting total MPnanodisc population.

We show that our model protein, recombinant histagged rhodopsin, can be transferred directly and with good recovery from host membranes into MSP nanodiscs through brief exposure to detergent in the presence of MSP and some extra lipid, followed by rapid extraction of detergent via $\beta$-cyclodextrin inclusion. Exposure to detergent can be limited to as brief as $60 \mathrm{~min}$. This approach, which strongly reduces the risk of detergent-induced irreversible loss of structural and/or functional integrity, is in principle applicable to any recombinant membrane protein.

Furthermore, we demonstrate that, even if both the target-protein and the MSP are his-tagged, extensive purification of the target-protein nanodiscs can be accomplished using a single IMAC step, removing over 95\% of contaminating protein, with high recovery of target-protein nanodiscs. This affords a level of purification that is sufficient for spectral and other functional studies. We demonstrate in this way, purified rhodopsin-nanodisc preparation, which upon illumination generates the active photointermediate Meta II, and can transduce the activation signal to its downstream signaling partner.

If required, high-level purification of the target-protein nanodiscs can be realized by a variety of established techniques, such as size-exclusion or ion-exchange chromatography, velocity or density centrifugation, or more dedicated methods like ligand- or immuno-affinity chromatography [e.g., Inagaki et al. (2013)].

Our approach provides a mild and generic platform for rapidly assembling membrane proteins into a soluble unit with maintenance of structural stability and functionality. This will be particularly useful for GPCRs as well as other integral membrane proteins that are relative sensitive to perturbation by detergent action. Here we demonstrate that this approach works successfully for rhodopsin. It remains to be shown that it is equally well applicable to potentially less stable membrane proteins. This, however, is better left to research groups with the required expertise with such proteins and outside the scope of this paper. With this paper, we trust to have laid a foundation for further research into this promising direction.

\section{Materials and methods}

\section{Materials}

Penicillin and streptomycin were from Gibco-BRL (Breda, The Netherlands). Leupeptin was obtained from MP Biomedicals (Santa Ana, CA, USA), heptakis (2,6-di-O-methyl)- $\beta$-cyclodextrin ( $\beta$-cyclodextrin) and asolectin from Sigma-Aldrich Co. (St. Louis, MO, USA). Grace's and Insect-Xpress insect media were from Lonza (Breda, The Netherlands); 11-cis retinal was a generous gift from Dr. Rosalie Crouch (Medical University of South Carolina, Charleston, SC, USA) through financial support from NEI; DDM and $n$-nonyl- $\beta$-glucoside (NG) were obtained from Serva Electrophoresis $\mathrm{GmbH}$ (Heidelberg, Germany). The plasmid for ZAP1 (Banerjee et al., 2008) was provided by Dr. Thomas Sakmar (The Rockefeller University, New York, NY, USA). The plasmids for MSP1D1 and MSP1E3D1 (Bayburt et al., 2002) were acquired through Addgene (Cambridge, MA, USA). Ni-NTA Superflow beads were purchased from Qiagen Benelux B.V. (Venlo, The Netherlands) and the high affinity analog of the Gt $\alpha$ C-terminal peptide $\left(\mathrm{NH}_{2}-\mathrm{VLEDLKSCGLF}-\mathrm{COOH}\right)$ from United Peptide Corporation (Chantilly, VA, USA).

Buffer A: $40 \mathrm{~mm}$ Tris, $0.3 \mathrm{~m} \mathrm{NaCl}$, and $1 \mathrm{~mm} \mathrm{NaN3}$ (pH 8.0). Buffer B: $20 \mathrm{~mm}$ Bis/Tris propane, $5 \mathrm{~mm} \mathrm{MgCl}, 1 \mathrm{~mm}$ dithioerythritol (DTE), $1 \mathrm{~mm}$ benzamidine, and $5 \mu \mathrm{m}$ leupeptin (pH 7.6). Buffer C: $20 \mathrm{~mm}$ Bis/ Tris propane, $1 \mathrm{~m} \mathrm{NaCl}, 1 \mathrm{~mm}$ histidine, and $5 \mu \mathrm{m}$ leupeptin ( $\mathrm{pH}$ 7.2). Buffer D: buffer B+100 mM NG.

\section{Construction of recombinant baculovirus}

Bovine opsin cDNA was ligated into the transfer vector pAcGP67 (BD Biosciences Clontech, Palo Alto, CA, USA). With the resulting vector recombinant baculovirus was generated, as described (Shirzad-Wasei et al., 2013). For relevant details, see the Supplementary Information. 


\section{Sf9 cell culture and rhodopsin expression}

Amplification of the rhodopsin-baculovirus and large-scale production of rhodopsin were performed as described (Klaassen and DeGrip, 2000; Shirzad-Wasei et al., 2013). Relevant details are given in the Supplementary Information. The culture was infected with rhodopsin-baculovirus at a multiplicity of infection $=0.1$. The cells were routinely harvested at 4 days post infection (dpi) and directly processed for regeneration with 11-cis retinal.

\section{Overexpression and purification of membrane scaffold proteins (MSP)}

ZAP1, MSP1D1, and MSP1E3D1 were overexpressed in the BL21(DE3)Rosetta2 Escherichia coli strain and purified over a Ni-NTA matrix using their N-terminal his-tag as described (Pandit et al., 2011). The yield of purified MSP varied between 60 and $200 \mathrm{mg} / \mathrm{l}$ culture. According to immunoblot analysis, the purified ZAP1 consisted of two major bands and a minor lower band between 25 and $30 \mathrm{kDa}$ (Figure 2), that all are competent in nanodisc formation (Pandit et al., 2011). The purified MSP1D1 and MSP1E3D1 consisted of a single band at ca 25 and ca $30 \mathrm{kDa}$, respectively. Purified MSPs were concentrated on a $10 \mathrm{~K}$ filter (Amicon ${ }^{\circledR}$ Ultra-15; EMD-Millipore, Billerica, MA, USA) and mixed with 1 volume of buffer A containing $5 \mathrm{~mm}$ DDM to a final concentration of $10-20 \mathrm{mg} / \mathrm{ml}$.

\section{Regeneration of opsin with ligand}

At $4 \mathrm{dpi}$, the infected $\mathrm{S} f 9$ cells were collected by centrifugation (10 min, $3000 \mathrm{~g}, 4^{\circ} \mathrm{C}$ ) and a membrane suspension was prepared as described (Shirzad-Wasei et al., 2013) in buffer $\mathrm{C}$ at a density equivalent to $10^{8}$ cells $/ \mathrm{ml}$ (see the Supplementary Information for full details). All subsequent manipulations were performed under dim red light ( $\lambda>620$ nm, RG620 cut-off filter; Schott, Menden, Germany). The ligand 11-cis retinal was added as a concentrated solution in dimethylformamide (30 $\mu \mathrm{m}$ final concentration) and the suspension was incubated for $1-2 \mathrm{~h}$ with constant rotation at RT. Subsequently, hydroxylamine was added from a $1 \mathrm{M}$ solution ( $\mathrm{pH}$ 7) to $50 \mathrm{~mm}$ final concentration. The membrane suspension was then directly used for solubilization of the components and nanodisc assembly.

\section{Formation of nanodiscs}

A membrane suspension, equivalent to $10^{8}$ cells $/ \mathrm{ml}$, containing regenerated rhodopsin, was diluted with buffer $\mathrm{C}$ containing $20 \mathrm{~mm}$ DDM to a density equivalent to $20 \times 10^{6}$ cells $/ \mathrm{ml}$ and mixed properly. After incubation under rotation at $4^{\circ} \mathrm{C}$ for maximally $1 \mathrm{~h}$, the insoluble material was removed by centrifugation $\left(30 \mathrm{~min}, 100000 \mathrm{~g}, 4^{\circ} \mathrm{C}\right.$ ). Asolectin was dissolved in buffer $\mathrm{D}$ and added to the solubilized membrane constituents at a 1:4 $(\mathrm{w} / \mathrm{w})$ ratio to total membrane protein, that is, $60 \mu \mathrm{g}$ per $10^{6}$ cells. This mixture was incubated for $15 \mathrm{~min}$ under constant rotation at $4^{\circ} \mathrm{C}$. ZAP1 was added in a molar ratio of 2:1 to total membrane protein, that is, $8 \mathrm{nmol}$ of ZAP1 per $10^{6}$ cells harvested, followed by incubation for maximally $30 \mathrm{~min}$ at $4^{\circ} \mathrm{C}$ under constant rotation. Nanodiscs were then formed through extraction of the detergent by addition of heptakis-(2,6-di- $O$-methyl)- $\beta$-cyclodextrin (DeGrip et al., 1998). Solid $\beta$-cyclodextrin was added in three steps to a final molar ratio of 1.2:1 $\beta$-cyclodextrin to detergent. Every addition of $\beta$-cyclodextrin was followed by incubation for $15 \mathrm{~min}$ at $4^{\circ} \mathrm{C}$ under constant rotation. Finally, insoluble material was pelleted (100 $000 \mathrm{~g}$, $30 \mathrm{~min}, 4^{\circ} \mathrm{C}$ ). The collected supernatant was directly used for purification or stored at $-80^{\circ} \mathrm{C}$ until further use.

\section{Purification of rhodopsin-nanodiscs}

His-tagged rhodopsin-nanodiscs were partially purified by batchwise chromatography over Ni-NTA agarose beads pre-equilibrated with buffer $\mathrm{C}$ containing $0.5 \mathrm{~mm}$ histidine (details are given in the Supplementary Information). All eluted fractions were assayed by SDS-PAGE and silver-staining or by quantification via immunoblotting, as described (Shirzad-Wasei et al., 2013). Fractions containing rhodopsin-nanodiscs were stored at $-80^{\circ} \mathrm{C}$.

\section{UV/Vis spectroscopy and photolysis}

UV/Vis spectroscopy was performed on a Lambda 18 spectrophotometer (Perkin Elmer, Norwalk, CT, USA). To quantify rhodopsin, spectra were recorded before and after illumination (150 W halogen light with fiber optics, $30 \mathrm{~s}$, Schott OG350 cut-off filter) in the presence of $50 \mathrm{~mm}$ hydroxylamine to convert all rhodopsin photoproducts into retinaloxime (Hubbard et al., 1971). If functional rhodopsin is present, difference spectra ('dark' spectrum subtracted from the spectrum after illumination) show a negative band around $500 \mathrm{~nm}$, representing the photolysed rhodopsin, and a positive band around $365 \mathrm{~nm}$ representing the retinaloxime formed upon illumination.

The late photointermediates Meta I and Meta II (Wald, 1968) were generated by brief illumination (10 s, Schott OG530 cut-off filter) at $10^{\circ} \mathrm{C}$ using buffer $\mathrm{B}$ at $\mathrm{pH} 8.0$ and $\mathrm{pH} 6.0$, respectively. To mimic interaction with the cognate $\mathrm{G}$ protein transducin (Gt) that only binds to Meta II, the high affinity analog of the C-terminal peptide of the $\mathrm{Gt} \alpha$-subunit was used ( $\left.\mathrm{NH}_{2}-\mathrm{VLEDLKSCGLF}-\mathrm{COOH}\right)$. This was added to the rhodopsin-nanodisc samples at $20 \mu \mathrm{M}$ final concentration just before the assay. A 'dark' spectrum was recorded and then photolysis was triggered by illumination for $10 \mathrm{~s}$ followed by immediately recording the first spectrum after illumination. Subsequently, spectra were recorded every $3 \mathrm{~min}$. After $30 \mathrm{~min}$, hydroxylamine was added to a final concentration of $50 \mathrm{~mm}$ and the presence of remaining rhodopsin was checked via 5 min of illumination and a final recording. This protocol was executed under four conditions, namely at $\mathrm{pH} 6.0$ and at $\mathrm{pH}$ 8.0, and with and without peptide. In order to clearly display the various transitions, difference spectra were generated by subtracting the 'dark' spectrum from the first spectrum after illumination, and the latter from the spectrum recorded $30 \mathrm{~min}$ after illumination.

\section{Dynamic light scattering}

The size distribution in the purified nanodisc samples was analyzed by dynamic light scattering (DLS) using the ZetasizerNanozS 
(Malvern Instruments Ltd, Malvern, UK), equipped with a $633 \mathrm{~nm}$ laser. If required, the rhodopsin-nanodisc samples were concentrated on a $10 \mathrm{~K}$ filter (EMD-Millipore). The DLS signals were analyzed by the internal software of the equipment and converted into a size distribution with scatter intensity.

Acknowledgments: We thank Dr. Thomas Sakmar (The Rockefeller University, New York, NY, USA) for providing the plasmid for ZAP1-expression. We acknowledge Dr. Rosalie Crouch (Medical University of South Carolina, Charleston, SC, USA) for a generous gift of 11-cis retinal through financial support from NEI. We thank Dr. Vincent Lemieux (Radboud University, Nijmegen, The Netherlands) for support with the DLS assay. This research was supported by funds to WJDG from the Council for Chemical Sciences of the Netherlands Organization for Scientific Research (NWO-CW project 700.54.008).

\section{References}

Aris, L., Gilchrist, A., Rens-Domiano, S., Meyer, C., Schatz, P.J., Dratz, E.A., and Hamm, H.E. (2001). Structural requirements for the stabilization of metarhodopsin II by the C-terminus of the $\alpha$ subunit of transducin. J. Biol. Chem. 276, 2333-2339.

Banerjee, S., Huber, T., and Sakmar, T.P. (2008). Rapid incorporation of functional rhodopsin into nanoscale apolipoprotein bound bilayer (NABB) particles. J. Mol. Biol. 377, 1067-1081.

Bayburt, T.H., Grinkova, Y.V., and Sligar, S.G. (2002). Self-assembly of discoidal phospholipid bilayer nanoparticles with membrane scaffold proteins. Nano Lett. 2, 853-856.

Bayburt, T.H., Grinkova, Y.V., and Sligar, S.G. (2006). Assembly of single bacteriorhodopsin trimers in bilayer nanodiscs. Arch. Biochem. Biophys. 450, 215-222.

Bayburt, T.H., Leitz, A.J., Xie, G., Oprian, D.D., and Sligar, S.G. (2007). Transducin activation by nanoscale lipid bilayers containing one and two rhodopsins. J. Biol. Chem. 282, 14875-14881.

Bayburt, T.H., Vishnivetskiy, S.A., Mclean, M.A., Morizumi, T., Huang, C.-C., Tesmer, J.J.G., Ernst, O.P., Sligar, S.G., and Gurevich, V.V. (2011). Monomeric rhodopsin is sufficient for normal rhodopsin kinase (GRK1) phosphorylation and arrestin-1 binding. J. Biol. Chem. 286, 1420-1428.

Blanchette, C.D., Segelke, B.W., Fischer, N., Corzett, M.H., Kuhn, E.A., Cappuccio, J.A., Benner, W.H., Coleman, M.A., Chromy, B.A., Bench, G., et al. (2009). Characterization and purification of polydisperse reconstituted lipoproteins and nanolipoprotein particles. Int. J. Mol. Sci. 10, 2958-2971.

Boldog, T., Grimme, S., Li, M., Sligar, S.G., and Hazelbauer, G.L. (2006). Nanodiscs separate chemoreceptor oligomeric states and reveal their signaling properties. Proc. Natl. Acad. Sci. USA 103, 11509-11514.

Bosman, G.J.C.G.M., VanOostrum, J., Breikers, G., Bovee-Geurts, P.H.M., Klaassen, C.H.W., and DeGrip, W.J. (2003). Functional expression of his-tagged rhodopsin in Sf9 insect cells. Methods Mol. Biol. 228, 73-86.

Chromy, B.A., Arroyo, E., Blanchette, C.D., Bench, G., Benner, H., Cappuccio, J.A., Coleman, M.A., Henderson, P.T., Hinz, A.K.,
Kuhn, E.A., et al. (2007). Different apolipoproteins impact nanolipoprotein particle formation. J. Am. Chem. Soc. 129, $14348-14354$.

Civjan, N.R., Bayburt, T.H., Schuler, M.A., and Sligar, S.G. (2003). Direct solubilization of heterologously expressed membrane proteins by incorporation into nanoscale lipid bilayers. Biotechniques 35, 556-563.

D’Antona, A.M., Xie, G., Sligar, S.G., and Oprian, D.D. (2014). Assembly of an activated rhodopsin-transducin complex in nanoscale lipid bilayers. Biochemistry 53, 127-134.

Daemen, F.J.M., Borggreven, J.M.P.M., and Bonting, S.L. (1970). Molar absorbance of cattle rhodopsin. Nature 227, 1259-1260.

DeCaluwé, G.L.J. and DeGrip, W.J. (1996). Point mutations in bovine opsin can be classified in four groups with respect to their effect on the biosynthetic pathway of opsin. Biochem. J. 320, 807-815.

DeCaluwé, G.L.J., VanOostrum, J., Janssen, J.J.M., and DeGrip, W.J. (1993). In vitro synthesis of bovine rhodopsin using recombinant baculovirus. Methods Neurosci. 15, 307-321.

DeGrip, W.J. (1982). Thermal stability of rhodopsin and opsin in some novel detergents. Methods Enzymol. 81, 256-265.

DeGrip, W.J. and Daemen, F.J.M. (1982). Sulfhydryl chemistry of rhodopsin. Methods Enzymol. 81, 223-236.

DeGrip, W.J., Daemen, F.J.M., and Bonting, S.L. (1980). Isolation and purification of bovine rhodopsin. Methods Enzymol. 67, 301-320.

DeGrip, W.J., VanOostrum, J., and Bovee-Geurts, P.H.M. (1998). Selective detergent-extraction from mixed detergent/lipid/protein micelles, using cyclodextrin inclusion compounds: a novel generic approach for the preparation of proteoliposomes. Biochem. J. 330, 667-674.

Denisov, I.G. and Sligar, S.G. (2011). Cytochromes P450 in nanodiscs. Biochem. Biophys. Acta 1814, 223-229.

Emeis, D., Kühn, H., Reichert, J., and Hofmann, K.P. (1982). Complex formation between metarhodopsin II and GTP-binding protein in bovine photoreceptor membranes leads to a shift of the photoproduct equilibrium. FEBS Lett. 143, 29-34.

Gohon, Y. and Popot, J.L. (2003). Membrane protein-surfactant complexes. Curr. Opin. Colloid Interface Sci. 8, 15-22.

Groenendijk, G.W.T., DeGrip, W.J., and Daemen, F.J.M. (1980). Quantitative determination of retinals with complete retention of their geometric configuration. Biochem. Biophys. Acta 617, 430-438.

Gulati, S., Jamshad, M., Knowles, T.J., Morrison, K.A., Downing, R., Cant, N., Collins, R., Koenderink, J.B., Ford, R.C., Overduin, M., et al. (2014). Detergent-free purification of ABC (ATP-bindingcassette) transporters. Biochem. J. 461, 269-278.

Hofmann, K.P. (1986). Photoproducts of rhodopsin in the disc membrane. Photobiochem. Photobiophys. 13, 309-327.

Hubbard, R., Brown, P.K., and Bownds, M.D. (1971). Methodology of vitamin A and visual pigments. Methods Enzymol. 18, 615-653.

Inagaki, S., Ghirlando, R., and Grisshammer, R. (2013). Biophysical characterization of membrane proteins in nanodiscs. Methods 59, 287-300.

Jamshad, M., Lin, Y.P., Knowles, T.J., Parslow, R.A., Harris, C., Wheatley, M., Poyner, D.R., Bill, R.M., Thomas, O.R., Overduin, M., et al. (2011). Surfactant-free purification of membrane proteins with intact native membrane environment. Biochem. Soc. Trans. 39, 813-818. 
Janssen, J.J.M., Mulder, W.R., DeCaluwé, G.L.J., Vlak, J.M., and DeGrip, W.J. (1991). In vitro expression of bovine opsin using recombinant baculovirus: the role of glutamic acid (134) in opsin biosynthesis and glycosylation. Biochem. Biophys. Acta 1089, 68-76.

Janssen, J.J.M., Bovee-Geurts, P.H.M., Merkx, M., and DeGrip, W.J. (1995). Histidine tagging both allows convenient single-step purification of bovine rhodopsin and exerts ionic strengthdependent effects on its photochemistry. J. Biol. Chem. 270, 11222-11229.

Klaassen, C.H.W. and DeGrip, W.J. (2000). Baculovirus expression system for expression and characterization of functional recombinant visual pigments. Methods Enzymol. 315, 12-29.

Klaassen, C.H.W., Bovee-Geurts, P.H.M., DeCaluwé, G.L.J., and DeGrip, W.J. (1999). Large-scale production and purification of functional recombinant bovine rhodopsin using the baculovirus expression system. Biochem. J. 342, 293-300.

Leitz, A.J., Bayburt, T.H., Barnakov, A.N., Springer, B.A., and Sligar, S.G. (2006). Functional reconstitution of $\beta_{2}$-adrenergic receptors utilizing self-assembling Nanodisc technology. Biotechniques 40, 601-610.

Long, A.R., O’Brien, C.C., Malhotra, K., Schwall, C.T., Albert, A.D., Watts, A., and Alder, N.N. (2013). A detergent-free strategy for the reconstitution of active enzyme complexes from native biological membranes into nanoscale discs. BMC Biotechnol. 13, 41.

Marty, M.T., Wilcox, K.C., Klein, W.L., and Sligar, S.G. (2013). Nanodisc-solubilized membrane protein library reflects the membrane proteome. Anal. Bioanal. Chem. 405, 4009-4016.

Matthews, R.G., Hubbard, R., Brown, P.K., and Wald, G. (1963). Tautomeric forms of metarhodopsin. J. Gen. Physiol. 47, 215-240.

Mors, K., Roos, C., Scholz, F., Wachtveitl, J., Dotsch, V., Bernhard, F., and Glaubitz, C. (2013). Modified lipid and protein dynamics in nanodiscs. Biochem. Biophys. Acta 1828, 1222-1229.

Navratilova, I., Sodroski, J., and Myszka, D.G. (2005). Solubilization, stabilization, and purification of chemokine receptors using biosensor technology. Anal. Biochem. 339, 271-281.

Orwick-Rydmark, M., Lovett, J.E., Graziadei, A., Lindholm, L., Hicks, M.R., and Watts, A. (2012). Detergent-free incorporation of a seven-transmembrane receptor protein into nanosized bilayer Lipodisq particles for functional and biophysical studies. Nano Lett. 12, 4687-4692.

Palczewski, K., Kumasaka, T., Hori, T., Behnke, C.A., Motoshima, H., Fox, B.A., Le Trong, I., Teller, D.C., Okada, T., Stenkamp, R.E., et al. (2000). Crystal structure of rhodopsin: a G protein-coupled receptor. Science 289, 739-745.

Pandit, A., Shirzad-Wasei, N., Wlodarczyk, L.M., van Roon, H., Boekema, E.J., Dekker, J.P., and DeGrip, W.J. (2011). Assembly of the major light-harvesting complex II in lipid nanodiscs. Biophys. J. 101, 2507-2515.

Park, S.H., Berkamp, S., Cook, G.A., Chan, M.K., Viadiu, H., and Opella, S.J. (2011). Nanodiscs versus macrodiscs for NMR of membrane proteins. Biochemistry 50, 8983-8985.

Popot, J.-L. (2010). Amphipols, nanodiscs, and fluorinated surfactants: three non-conventional approaches to studying membrane proteins in aqueous solutions. Annu. Rev. Biochem. 79, 737-775.

Prior, M.J., Larance, M., Lawrence, R.T., Soul, J., Humphrey, S., Burchfield, J., Kistler, C., Davey, J.R., La-Borde, P.J., Buckley, M., et al. (2011). Quantitative proteomic analysis of the adipocyte plasma membrane. J. Proteome. Res. 10, 4970-4982.
Ratnala, V.R.P., Swarts, H.G.P., VanOostrum, J., Leurs, R., de Groot, H.J.M., Bakker, R.A., and DeGrip, W.J. (2004). Large-scale overproduction, functional purification and ligand affinities of the His-tagged human histamine $\mathrm{H} 1$ receptor. Eur. J. Biochem. 271, 2636-2646.

Rigaud, J.-L., Lévy, D., Mosser, G., and Lambert, O. (1998). Detergent removal by non-polar polystyrene beads - applications to membrane protein reconstitution and two-dimensional crystallization. Eur. Biophys. J. Biophys. Lett. 27, 305-319.

Ritchie, T.K., Grinkova, Y.V., Bayburt, T.H., Denisov, I.G., Zolnerciks, J.K., Atkins, W.M., and Sligar, S.G. (2009). Reconstitution of membrane proteins in phospholipid bilayer nanodiscs. Methods Enzymol. 464, 211-231.

Rosenbusch, J.P. (2001). Stability of membrane proteins: relevance for the selection of appropriate methods for high-resolution structure determinations. J. Struct. Biol. 136, 144-157.

Sato, K., Morizumi, T., Yamashita, T., and Shichida, Y. (2010). Direct observation of the $\mathrm{pH}$-dependent equilibrium between metarhodopsins I and II and the $\mathrm{pH}$-independent interaction of metarhodopsin II with transducin C-terminal peptide. Biochemistry $49,736-741$.

Shirzad-Wasei, N., VanOostrum, J., Bovee-Geurts, P.H., Wasserman, M., Bosman, G.J., and DeGrip, W.J. (2013). Large scale expression and purification of mouse melanopsin-L in the baculovirus expression system. Protein Expr. Purif. 91, 134-146.

Silvius, J.R. (1992). Solubilization and functional reconstitution of biomembrane components. Annu. Rev. Biophys. Biomol. Struct. 21, 323-348.

Sone, N., Yoshida, M., Hirata, H., and Kagawa, Y. (1977). Reconstitution of vesicles capable of energy transformation from phospholipids and adenosine triphosphatase of a thermophilic bacterium. J. Biochem. 81, 519-528.

Swainsbury, D.J., Scheidelaar, S., VanGrondelle, R., Killian, J.A., and Jones, M.R. (2014). Bacterial reaction centers purified with styrene maleic acid copolymer retain native membrane functional properties and display enhanced stability. Angew. Chem. Int. Ed. Engl. 53, 11803-11807.

Tsukamoto, H., Szundi, I., Lewis, J.W., Farrens, D.L., and Kliger, D.S. (2011). Rhodopsin in nanodiscs has native membrane-like photointermediates. Biochemistry 50, 5086-5091.

VanBreugel, P.J.G.M., Bovee-Geurts, P.H.M., Bonting, S.L., and Daemen, F.J.M. (1979). Biochemical aspects of the visual process $\mathrm{XL}$. Spectral and chemical analysis of metarhodopsin III in photoreceptor membrane suspensions. Biochem. Biophys. Acta 557, 188-198.

Vogel, R., Siebert, F., Zhang, X.Y., Fan, G.-B., and Sheves, M. (2004). Formation of meta III during the decay of activated rhodopsin proceeds via meta I and not via meta II. Biochemistry 43 , 9457-9466.

Wald, G. (1968). The molecular basis of visual excitation. Nature 219, 800-807.

Wallin, E. and VonHeijne, G. (1998). Genome-wide analysis of integral membrane proteins from eubacterial, archaean, and eukaryotic organisms. Protein Sci. 7, 1029-1038.

Whorton, M.R., Bokoch, M.P., Rasmussen, S.G.F., Huang, B., Zare, R.N., Kobilka, B.K., and Sunahara, R.K. (2007). A monomeric G protein-coupled receptor isolated in a high-density lipoprotein particle efficiently activates its G protein. Proc. Natl. Acad. Sci. USA 104, 7682-7687.

Whorton, M.R., Jastrzebska, B., Park, P.S.H., Fotiadis, D., Engel, A., Palczewski, K., and Sunahara, R.K. (2008). Efficient coupling of 
transducin to monomeric rhodopsin in a phospholipid bilayer. J. Biol. Chem. 283, 4387-4394.

Wishart, D.S., Knox, C., Guo, A.C., Shrivastava, S., Hassanali, M., Stothard, P., Chang, Z., and Woolsey, J. (2006). DrugBank: a comprehensive resource for in silico drug discovery and exploration. Nucleic Acids Res. 34, D668-D672.

Zhou, H.X. and Cross, T.A. (2013). Influences of membrane mimetic environments on membrane protein structures. Annu. Rev. Biophys. 42, 361-392.
Zoonens, M., Comer, J., Masscheleyn, S., Pebay-Peyroula, E., Chipot, C., Miroux, B., and Dehez, F. (2013). Dangerous liaisons between detergents and membrane proteins. The case of mitochondrial uncoupling protein 2. J. Am. Chem. Soc. 135, 15174-15182.

Supplemental Material: The online version of this article (DOI: 10.1515/hsz-2015-0100) offers supplementary material, available to authorized users. 